\title{
Short Version of 'The Brain, Explained': A Response Process Theory of Brain Function
}

\author{
Ari Rappoport \\ The Hebrew University of Jerusalem, Israel \\ ari.rappoport@mail.huji.ac.il \\ www.cs.huji.ac.il/ arir
}

\begin{abstract}
Understanding brain function is one of the most important open problems in science today. At present, there is no concrete theory for how the brain works. Here, a theory is presented that provides a detailed mechanistic biological account of the brain's capacities. Brain function is managed by a response $(\mathrm{R})$ process that is structurally similar to the immune response, and shows anatomical and molecular specificity. Different $\mathrm{R}$ process stages utilize different cortical layers, hippocampus fields, basal ganglia paths, GABAergic interneurons, cerebellum paths, and molecular agents such as dopamine, serotonin and opioids. We show how the $\mathrm{R}$ process supports hierarchical action sequences, language and thought. The theory is supported by a large body of experimental evidence in many modalities, and accounts for virtually all of the major facts known about the brain at the system level.
\end{abstract}

Keywords. Neuroscience, basal ganglia, cortical layers, cerebellum, habenula, inhibition, predictions, dopamine, serotonin, acetylcholine, opioids, mirror neurons, place cells, language, imagery, working memory.

\section{Introduction}

How the brain works is one of the major open problems in science today. Following Newton, Einstein, Darwin and many others, we understand many aspects of the physical world and life. However, we do not understand how living organisms act and think. These capacities are known to be mediated by the brain, but currently there exists no brain theory or anything close to it [Grillner, 2014], despite the enormous amount of invested research effort.

This situation is not surprising, given that a theory of brain function needs to explain many different phenomena, including motor control, perception, language and imagery. Indeed, some researchers think that we may never understand how the human brain works [Adolphs, 2015], or that it would take decades [Koch and Marcus, 2014] and require large concerted data collection projects.

This paper suggests that such pessimism is not warranted, presenting a theory of brain function that explains virtually all of the important phenomena. The main thesis is that 




Figure 1: The R process (general version). Arrows: excitatory flow. Thick line: coarse response. Hollow circle: candidate focusing. Dotted line: response execution affects incoming sensory inputs.

brain operation is governed by a well-defined response $(\mathrm{R})$ process, which is strongly reflected in the brain's anatomy and molecular physiology and is structurally similar to the immune response. The theory (completed in 2017 and denoted by R17) is supported by a large amount of experimental evidence in many modalities. It is mechanistic and refutable, and is very different from the existing standard accounts in most sub-areas.

The paper presents the theory and summarizes its supporting evidence. Details given in the long version of the paper [Rappoport, 2018] are marked by ${ }^{D}$. Plasticity and disorders (including glia, sleep, etc.) undergo different processes and are described elsewhere.

\section{The Theory}

\subsection{The $R$ process}

The brain's role is to map sensory inputs (internal and external) to movement responses. Brain operation is managed by a response $(\mathbf{R})$ process having several stages ( $R$ modes). The $\mathrm{R}$ process distinguishes between innate responses, present in all healthy members of a species, and adaptive responses, which are organism-specific. There are two prototypical flow directions, from inputs to responses (bottom-up, BU), and vice versa (top-down, TD).

The process starts with BU sensory inputs (Figure 1). If there is an innate response, it is executed. Otherwise, if there is an adaptive learned response, it is executed. Otherwise, the brain starts an adaptive acute response having two $\mathrm{R}$ modes. The alert mode induces widespread BU excitation and recruits energy resources. Alerts are answered by a TD decision making (DM) mode comprised of candidate generation and competition. Candidates include previously learned responses responding to partial features of the current input. This can yield a rapid coarse adaptive response. Competition between candidates results in a focused adaptive response. Response execution utilizes innate capacities. The responses involved in execution are learned via plasticity processes, to facilitate their future activation.

At this level of description, the $\mathrm{R}$ process is basically identical to the immune response (see below).

The brain's R process includes several additional modes. Each mode is supported by 




Figure 2: The brain's R process. Arrows: excitation. T-junctions: suppression. Rgens are shown in italics. Thick rectangles: modes participating in both the $\mathrm{N}$ process and the $\mathrm{Q}$ process. Thick line: coarse response.

All modes are implemented by dedicated anatomical parts of the brain, with some overlap. See R networks section. All modes are promoted by specific Rgens (except sensory inputs). ACh: acetylcholine (nAChR: nicotinic receptors, mAChR: muscarinic receptors). AVP: arginine vasopressin. BEND: beta-endorphin. CRH: corticotropin-releasing hormone. DA: dopamine. DHEA: dehydroepiandrosterone. DYN: dynorphin. ECBs: endocannabinoids. ENK: enkephalin. GCs: glucocorticoids (cortisol, corticosterone). $\mathrm{MCH}$ : melanin-concentrating hormone. NE: norepinephrine. NOC: nociceptin. OT: oxytocin. OX: orexin (also called hypocretin). SER: serotonin. SP: substance P. BEND, DYN, ENK and NOC are opioids. Detailed planning circuits are not shown.

a dedicated part of the brain's anatomy and by dedicated molecular agents (Rgens) (Figure 2). This observation is the key contribution of R17 and what allows it to connect high-level cognitive notions to concrete experimental results.

First, an internal needs mode continuously conveys survival-related needs, including hunger, thirst, sex, minerals etc. This mode excites the brain circuits involved in the satisfaction of specific needs, prompting the formation of relevant movements.

Second, innate responses range from low-level ones such as muscle contraction by alpha motoneurons (aMTNs), reflexes, and the orienting of attention, to high-level ones such as fight, freeze and flight.

Third, there are two types of DM, acting and planning (or thinking), for urgent and non-urgent DM respectively. Acting is promoted by inputs having strong valence. Responses are disinhibited, and a vigorous coarse response can be induced without competition. During planning, movement responses are suppressed, and the brain considers response alternatives.

Competition is mediated by coordination networks (CNs), comprised of GABAergic 
inhibitory interneurons (IINs) in adaptive areas and GABAergic and glycinergic IINs in innate areas.

Fourth, to sustain goals and support response sequences, execution has two sub-modes, prediction (goal-setting) and response. Predictions include sensory goals of executing actions, general sensory predictions, and actions prepared for immediate execution. Responses include executing movements and perceived sensory objects (perception is a sensory response). Predictions represent the near future while responses represent the present.

Fifth, when execution encounters difficulties (e.g., due to critical surprises), a mistake mode rapidly switches from execution to planning.

Sixth, when alerting objects are identified as unthreatening, an interaction mode suppresses alerts to allow object exploration.

Finally, an extended alert mode initiated after alerts sustains alerts beyond their initial triggers, and summons an energy management mode comprised of two sub-modes. Continue promotes execution, while protection suppresses adaptive responses in order to protect cells from oxidative stress.

Rgens support their modes by exciting their networks and cooperating ones, and suppressing networks and Rgens supporting competing modes ${ }^{D}$. Notably, the role of opioids is termination of the $\mathrm{R}$ process by innate responses, each OP supporting a different prototypical innate response (consume, fight, freeze, flight).

The brain is best viewed as running two nested instances of the $\mathrm{R}$ process at different time scales. The seconds-to-hours need $(\mathbf{N})$ process addresses needs and their satisfaction (what needs to be done), while the millisecond scale quax $(\mathbf{Q})$ process supports execution (how things are done).

\subsection{R networks}

Each R mode uses a dedicated anatomical brain part (network).

The sensory network includes peripheral, spinal and proprioceptive sensory fibers, core neurons (parvalbumin (PV)-expressing excitatory neurons) in specific and non-specific thalamus nuclei ${ }^{D}$, cortical layer 4 (L4) excitatory neurons (L3 in agranular areas ${ }^{D}$ ), the corticothalamic neurons in L6, and the cerebellum climbing fibers.

The alert network includes cortical L3b (the bottom part of L3) pyramids, and NE nuclei (the locus coeruleus (LC) and brainstem adrenergic cell groups).

The DM network includes cortical L2/3 pyramids, the direct path of the basal ganglia (BG), hippocampus dentate gyrus, CA3 and CA2 pyramids, DA, SER and ACh nuclei, and the $\mathrm{DCN}^{1}$.

The prediction network includes the slender-tufted bilaterally projecting (to cortex and BG) cortical L5 pyramids ${ }^{D}$ (mainly L5a) (also L3 in agranular areas), the indirect BG path, hippocampus CA1 pyramids, cerebellum granule cells, gamma motoneurons ${ }^{D}$, and DA nuclei.

The response network includes aMTNs and excitatory innate circuits innervating them, the thick-tufted ipsilaterally projecting (to cortex, BG, thalamus, spinal cord, motor centers) cortical L5 pyramids ${ }^{D}$ (L5b), the indirect BG path, the thalamic matrix (calbindinexpressing excitatory neurons), the subiculum, cerebellum Purkinje cells and deep nuclei, the amygdala, ACh nuclei, and the ECN.

Cortex contains three GABAergic coordination networks $(\mathbf{C N s})^{D}$. The DM coordination network (DCN) contains Martinotti IINs expressing somatostatin (SOM) that target apical dendrites of response network neurons, vasoactive intestinal polypeptide (VIP)-

\footnotetext{
${ }^{1}$ See coordination networks below.
} 




Figure 3: Intra-node connections. Only the main patterns are shown. Filled squares: excitatory neurons. The thick (slender) tufted dendritic tree of a response (prediction) neuron is shown in thick (thin) dashed lines. Empty squares: coordination IINs. The execution coordination network $(\mathrm{ECN})$ is on the right in thick lines. ECN interconnections are shown without arrows to emphasize gap junctions. The DM coordination network (DCN) is on the left (s: SOMs, v: VIPs, n: NGFCs). Neurogliaform cells target all cell types, depicted by a single general arrow. The deep networks have subcortical projections.

expressing IINs that target SOM IINs, and L1 neurogliaform cells (NGFCs) that target all IINs and excitatory neurons. The execution coordination network (ECN) contains PV- and CCK-expressing basket cells that target cell somas. The response suppression network (RSN) contains PV-expressing chandelier cells that target the axon initial segment (AIS). IINs that do not express PV or SOM express SER3, a ionotropic SER receptor closely related to nicotinic ACh receptors (nAChRs, see below).

Other parts of the brain, including the BG, hippocampus, and amygdala, also contain an ECN and a DCN. All brain parts (including the thalamus, cerebellum, spinal cord, and other innate areas) contain an ECN.

The mistake network includes the lateral habenula, SER nuclei, and the RSN.

The amygdala is the interface between innate and adaptive circuits ${ }^{D}$. Its central complex controls innate responses, and its BLA complex learns the valence of sensory inputs (i.e., the type of innate response associated with the input). BLA strongly innervates the alert network in cortical valence areas (medial prefrontal cortex (mPFC), anterior cingulate cortex, orbitofrontal cortex).

The adaptive parts of the brain include cortex, BG, thalamus, hippocampus, cerebellum and amygdala BLA. The hippocampus represents place, object and valence combinations (i.e., events ${ }^{D}$, according with its famous role in episodic memory). Its $\mathrm{R}$ networks are organized by fields (indicated above) rather than layers ${ }^{D}$, probably to support the huge number of possible events and neurogenesis.

Cortex is arranged in nodes ${ }^{D}$ (microcolumns) and layers. The cortical networks show specific intra-node interconnection patterns (Figure 3), mainly sensory-alert-DM-predictionresponse and sensory-response. Across cortex, excitatory neurons in the same network are extensively interconnected, and the deep and superficial networks are interconnected via 
long-distance connections to allow sensory and action responses to generate alerts and candidates, respectively.

We say that an area, node or neuron $N$ represents ${ }^{D}$ an entity $E$ (external or internal object or action) if and only if $N$ is consistently activated when the brain responds to or executes $E$. Node neurons in the same network most likely represent the same entity. In practice, we can only determine the representational function of areas (e.g., using fMRI and lesions). We broadly distinguish between object, action and valence areas (edgeposterior, mid-anterior and medial cortex, respectively), and between low-level (motor M1 and premotor cortices) and high-level (SMA, preSMA, dlPFC, frontal pole) action areas.

The major adaptive circuit includes cortex, the basal ganglia (BG), and the thalamus (Figure 4). The BG and thalamus are two large bilateral sets of nuclei at the center of the brain. The thalamic core conveys BU flow onto the cortical sensory, alert and response networks. During acute responses, cortical response network neurons need sustained drive in order to fire continuously. Thalamic matrix neurons, which project diffusely to L1-3/5a, provide this drive and their recruitment is essential for sustained adaptive responses. These neurons (as well as other motor centers) are tonically suppressed by the output nuclei of the BG. Thus, the BG must be silenced or recruited to form adaptive responses.

The BG has two paths, a direct path from the input nucleus (the striatum, STR) to the output nuclei whose neurons express dopamine D1 receptors (D1Rs), and an indirect path utilizing additional nuclei (GPe, subthalamic nucleus (STN)) whose neurons express D2Rs. In R17, the D1 path is part of the DM network, supporting acting via disinhibition, while the D2 path and STN ${ }^{2}$ are part of the execution (prediction and response) networks ${ }^{3}$. The direct path initiates acutely-driven actions, and the indirect path executes them and supports automated actions (detailed below).

\subsection{The $Q$ process: motor control, thought, language}

The $\mathrm{Q}$ process is the fast (milliseconds) instance of the $\mathrm{R}$ process. It mediates action execution via the input, alert, DM, execution and innate networks.

Hierarchical sequences. We refer to the set of neurons participating in an active sensoryresponse mapping (i.e., response execution) as a quax ${ }^{4}$ (plural quacia). The quax (Q) process describes how neurons join and leave the quax (transitions).

The quax executing an action contains response neurons representing the action. This includes low-level action nodes innervating spinal circuits and alpha motoneurons, and higher-level nodes representing action plans. The quax also typically contains response neurons in valence areas representing the motivation for executing the action.

An important principle of $\mathrm{R} 17$ is that the quax also contains prediction network neurons in nodes representing the sensory goals of the action, which are crucial for implementing sequences.

When a neuron is active, it excites all of its neuronal targets. Since post-development connections in the brain exist only if they were used in past responses, when the brain executes an action, all of the neurons that have participated in executing similar past actions are excited (mobilized) to some extent. Mobilization is TD (reflecting responses) and occurs in the DM, prediction and response networks. When the goal of the action is attained, sensory flow reaches the nodes representing the goal and excites the node's sensory, response and alert networks. Since the node's response network is already excited by its prediction

\footnotetext{
${ }^{2}$ And cholinergic interneurons, see below.

${ }^{3}$ This account is dramatically different from the standard BG account, see discussion.

${ }^{4}$ The term is loosely derived from 'cooperating axes', referring to the BU and TD object-action flow axes.
} 


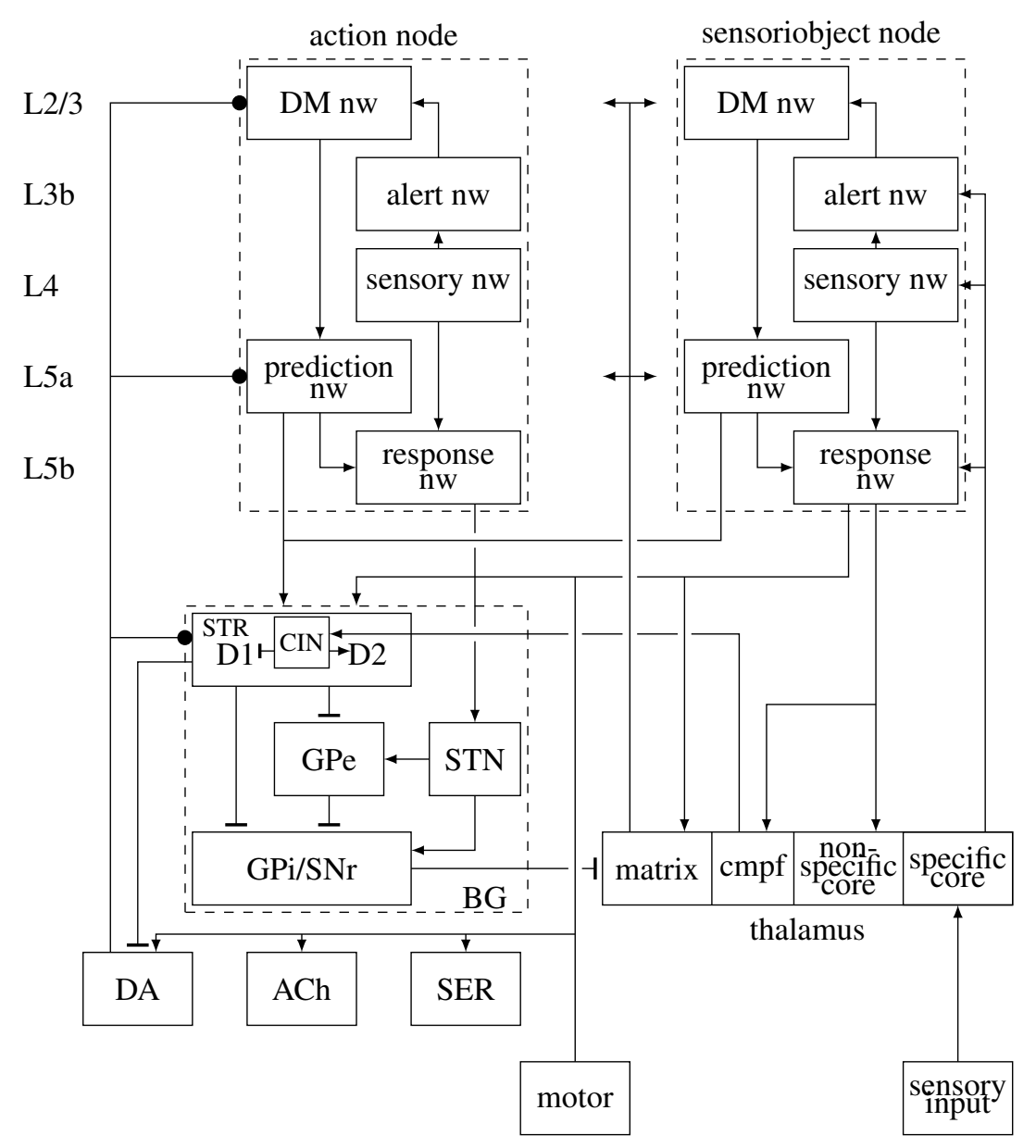

Figure 4: The major adaptive brain circuit, showing the BG, thalamus, two cortical columns (in primary sensory cortex (right) and frontal cortex (left)), and three Rgen nuclei. To avoid clutter, some connections and areas are not shown. Arrows, $\mathrm{T}$ junctions and circles indicate excitatory flow, GABAergic flow, and Rgen release respectively.

CIN: cholinergic interneurons. cmpf: centeromedian-parafascicular thalamic complex. D1 (D2): the STR projection neurons expressing D1R (D2R). GP: globus pallidus (i: internal, e: external). nw: network. SNr: subtantia nigra reticulata. STR: striatum. STN: subthalamic nucleus.

network, the combined BU (sensory) and TD (predictions) flow easily activates it. The activated response network sends BU flow to the DM and response networks in action areas via long-distance connections. When this flow meets a mobilized node, the combined BU and TD flow again activates its response network, thereby selecting the next action. Thus, executing responses mobilize many next-action candidates, and sensory reality selects which of them get executed.

High-level action nodes representing action plans do not need to specify the sequential order of execution. All they need is to excite all of the actions of the action plan, and execution order gets specified by sensory input. This way, hierarchical action sequences emerge via sensory-action interactions without the need for central management. This architecture also allows parallel action execution (e.g., when reaching to grasp an object, you move your fingers while extending your arm).

During sensory surprises, the alert and DM networks play a stronger part in selecting the next action (see below). 
Coordination. IINs are innervated by excitatory neurons, by IINs in the same network (often via gap junctions for rapid neurotransmission), and by IINs in other networks. We say that when a neuron fires, it sends coordination requests to its target IINs. The effect of IIN activation is not always inhibitory. When an IIN fires just before its target fires, it indeed suppresses it. However, when an IIN fires just after its target fires, it does not suppress it. Here, the IIN resets its targets' excitatory states, which gets their firing synchronized. IINs implement a winner-takes-all mechanism in which neurons whose activity accords with local flow are synchronized while others are silenced.

In quax transitions, it is common for sensory input to trigger coarse acting by activating several different response nodes (e.g., when predictions are not precise). Acting and competition are supported by the CNs. By VIP suppression of the SOMs, the DCN opens a disinhibitory 'hole' that allows the winning response to receive local and long-range inputs onto its dendrites [Karnani et al., 2016, Letzkus et al., 2015]. The hole is wide during acting and narrows following competition. NGFCs surround the hole with a 'blanket' of inhibition. The ECN sustains the winners via the rapid synchronizing action of basket cells.

Cerebellum. The cerebellum has a unique architecture supporting rapid response sequences via a $\mathrm{Q}$ process ${ }^{D}$. Predictions, represented by granule cells, innervate Purkinje cells (PCs) via parallel fibers, and sensory information arriving from the inferior olivary reaches PCs via climbing fibers. When predictions and reality converge on the same PC, it is briefly suppressed and the corresponding response is disinhibited ${ }^{D}$.

Language. The function of the brain is to generate movement. In many situations, it is desired to generate movements made by other organisms. To do this, the organism needs to activate neurons in other organisms' brains. This is the function of communication. Language is a communication medium that uses audio to bridge the inter-neuronal gap.

Language is implemented by a $\mathrm{Q}$ process similar to that implementing motor actions. The only difference is that the brain needs to acquire representations that mediate between its goals and the sounds that induce the other organism to move to satisfy these goals. The organism's goals are the meaning (semantics) of the linguistic utterance, the audio nodes support phonetics and phonology, and mediation is done by lexico-syntactic representations ${ }^{D}$.

Imagery (thought). Imagery can be visual (motor imagery) or auditory (thought, silent speech, music). It represents the response candidates activated in the DM planning mode.

Imagery is implemented by the standard $\mathrm{Q}$ process. The only difference between imagery and motor quacia is that the former contain action goals that are not represented by external sensory nodes. Transitions still occur after goal node activation in response mode, but they are not triggered by external sensory input. Instead, action nodes use cues that convey triangulating drive from several different directions ${ }^{D}$ (e.g., 'an animal whose name starts with " $p$ " uses two cues) $)^{5}$. This is possible when the brain can generate strong drive in the TD direction, as is the case with humans. Note that sensory nodes can sometimes endogenously activate in response mode, inducing hallucinations.

Automaticity. Automated responses are characterized by rapid triggering by sensory inputs and lack of acute ('emotional') responses. The brain uses precise predictions to immediately find the appropriate response, without having to propagate the input further. Hence, the response neuron is suppressed after it fires, via the high-affinity receptors of the Rgens involved in acute responses (D2R, alpha2-NER, alpha4beta2 nAChR, SER1/4/76), which

\footnotetext{
${ }^{5}$ Note that the cues should be activated earlier in the same manner.

${ }^{6}$ For readability, we use SER1 rather than 5-HT1 etc.
} 
are released in small amounts during automated responses. Most of these also suppress further Rgen release via autoreceptors.

Internal cognition. Consciousness and general awareness can be scientifically tackled via reporting ${ }^{D}$. Emotions are reports of $\mathrm{N}$ modes ${ }^{D}$, accessible to adaptive quacia via interoceptive sensory inputs continuously conveying the status of body tissues [Craig, 2014].

\subsection{The $\mathrm{N}$ process: functional states}

The need $(\mathrm{N})$ process is the brain's main instance of the $\mathrm{R}$ process. Here we elaborate on $\mathrm{N}$ modes and their mechanisms.

Alert. Alerts are typically triggered by sensory surprises, which trigger an innate orienting of attention (OOA) response, sensory network bursts, and NE release. NE recruits energy resources via the sympathetic autonomic nervous system, and widely excites the alert and DM networks. Bursts yield strong flow that activates the response network in many areas.

DM. Alerts excite the DM and execution networks in sensory and PFC areas. PFC execution neurons innervate movement and sensory response candidates via long-distance connections to the DM and prediction networks. They also excite the DCN. These connections supported past responses made to features of the current input, such that a large set of potentially relevant response candidates is activated. The DCN disinhibits response neurons, creating an acting hole that narrows down after competition.

In parallel, response neurons widely innervate Rgen nuclei, with sensoriobject, motor, and high-level action areas strongly innervating ACh, DA and SER nuclei respectively ${ }^{D}$. The Rgen neurons innervate cortex and the BG (especially DA) in a topographic manner.

DA acts through two receptors families, D1 and D2, which couple to Gs and Gi/o to support rapid firing and suppression, respectively. The STR contains cholinergic inhibitory neurons (CINs) that excite indirect MSNs and suppress direct MSNs. Surprises induce sensory network flow into thalamic cmpf neurons, which project to the CINs (Figure 4). The cmpf-induced CIN burst briefly suppresses execution ${ }^{7}$, and CINs are then silenced by afterhyperpolarization. Simultaneously, surprises yield a strong BG DA surge, which activates the high-affinity D2R on indirect path MSNs and CINs, suppressing ongoing execution. The cortical prediction and response networks both project to the STR (to both paths) ${ }^{D}$. The DA surge and the removal of CIN suppression let cortex freely activate the D1 MSNs, and this enabling of the acting mode disinhibits the thalamus and allows its recruitment by cortical response neurons.

Thus, the DM response to strong surprise consists of disinhibition in both cortex and the BG. The former involves a wide 'hole' disinhibiting response neurons, while the latter involves a wide channel (the direct path) that disinhibits the thalamus to allow competing neurons access to it. Competition is mediated by cortical and BG IINs. DA uptake in the STR is rapid, so when competition ends, the D2 suppression of MSNs is removed, letting them join the D1 winners. D1R activation has a long effect, but eventually the D1 MSNs stop firing, leaving the D2 MSNs support execution alone. Thus, the direct path is needed for acute response initiation, and the indirect path, which includes the D2 MSNs, STN and CINs, executes responses.

When the alerting input is very strong, vigorous coarse disinhibited responses are executed before competition is resolved or initiated. When alerts do not push for an immediate

\footnotetext{
${ }^{7}$ Indirect path execution depends on fine synchrony, so it is stopped by bursts.
} 
response, DM invokes planning, where movements and innate responses are suppressed, and imagery invokes various scenarios in order to find the most appropriate response.

Prediction. After competition resolution, DA sustains the winners' goals, via D1Rs on the prediction and DM networks [Arnsten, 2013]. Cortical uptake of DA is much slower than in the $\mathrm{BG}$, allowing long-term sustenance of decisions (motivation).

Execution. ACh supports disinhibition via alpha7 $\mathrm{nAChRs,} \mathrm{and} \mathrm{competition} \mathrm{and} \mathrm{execu-}$ tion via alpha4beta2 nAChRs and muscarinic (mAChR) receptors ${ }^{D}$. ACh also directly supports movement execution, being the neurotransmitter that induces muscle contraction. The competition focusing effect of $\mathrm{ACh}$, and the fact that it acts more strongly than DA in sensoriobject areas, explain its association with attention to stimuli [Sarter et al., 2016]. Execution is also supported via synchrony by the ECN.

Consume. The initial stages of food consumption and sex, and subsequent relaxation, are promoted by beta-endorphin (BEND), which acts via mu-opioid receptors (MORs). When consummatory acts are imminent, BEND promotes DA (to support acting) and suppresses adaptive TD responses (to disinhibit the innate circuits supporting consume, and to prevent interference by TD responses). As such, BEND is the primary agent supporting hedonic pleasure. After the act, BEND gradually suppresses innate circuits as well.

The initial action of BEND explains the euphoria-inducing effect of heroin, and its response suppression explains the role of morphine as a powerful analgesic. Both drugs act on MORs; morphine acts on other opioid receptors as well.

Food and sex also involve other agents, not described here.

Satisfaction. Oxytocin (OT) facilitates birth (parturition, lactation, maternal behavior), orgasm [Veening et al., 2015], and pleasant touch [Uvnäs-Moberg et al., 2014]. These are needs satisfied by conspecifics, explaining the well-known association of OT and sociality.

OT and BEND are both released during culmination of need satisfaction, signalling successful termination of the R process. BEND promotes movements produced by skeletal muscles, while OT affects smooth muscles.

Failure. The opioid dynorphin promotes freeze and withdrawal innates ${ }^{D}$. It is released locally by excessively firing neurons, including D1-expressing BG MSNs and central amygdala neurons, and suppresses them retrogradedly to protect neurons from oxidative stress and enable the brain to form a different response. The withdrawal role of DYN explains its involvement in depression [Tejeda et al., 2012].

Mistake. The habenula is located in the dorsomedial thalamus. The lateral habenula $(\mathrm{LHb})$ suppresses DA nuclei and is suppressed by $\mathrm{DA}^{D}$, and excites and is excited by DRN $\mathrm{SER}^{D}$. LHb is activated in motivated negative situations [Proulx et al., 2014], especially negative surprise. Its role is clearly to switch from action to planning.

The medial habenula is strongly excited by ACh to promote execution. The R17 role of the habenula as a whole is to stop or reinforce execution following (aversive) surprise.

The RSN (see CNs) promotes the mistake mode in cortex, strongly suppressing response neurons by targeting their AIS.

Other modes are discussed in ${ }^{D}$.

\section{Evidence}

R17 is supported by converging evidence from a range of modalities. Anatomical evidence from networks and connectivity patterns has been described above. Here we present evi- 
dence from molecular agents, EEG, electrophysiology, imaging, direct neural recording, and immunity.

\subsection{Molecular agents (Rgens)}

Needs. Internal needs directly excite hypothalamic (HT) neurons that release relevant Rgens. OX neurons are stimulated (inhibited) by low (increasing) glucose, and project to the VTA DA nucleus to induce acting [Sakurai, 2014]. OX is associated with the classical brain arousal system and promotes wakefulness.

AVP is associated with three needs, water, mating and aggression ${ }^{D}$. In all cases, AVP signals the need for making an energy-consuming physical effort to address external challenges. It is closely structurally related to oxytocin, which promotes fluid-related satisfaction. A unified view is that AVP and OT address the same needs, AVP before and OT during and after need satisfaction.

Alert. Surprises yield alerts by inducing bursts of action potentials (APs). For example, after hyperpolarization longer than $100 \mathrm{~ms}$, firing thalamic neurons activate T-type $\mathrm{Ca} 2+$ channels causing a low-threshold spike, on top of which can ride a series of high frequency APs [Sherman and Guillery, 2013].

NE acts via excitatory alpha1 and beta-NERs and inhibitory alpha2-NERs. Beta (alpha1) NERs couple to Gs (Gq), supporting immediate (extended) execution. Accordingly, beta-NERs are mostly expressed in the sensory and alert networks and in innate circuits, while alpha1-NERs are mostly expressed in the DM network and in HT CRH neurons [Goldman-Rakic et al., 1990]. Alpha2-NERs suppress NE release as autoreceptors, and suppress the response network, both directly and by closing $\mathrm{HCN}$ channels ${ }^{D}$.

DM. The role of cortical VIP and SOM neurons in competition is reviewed in [Karnani et al., 2016, Letzkus et al., 2015]. Supporting the R17 BG DM account, direct neurons activate before indirect ones when starting an attended action ${ }^{D}$, and both paths are used during everyday actions [Cui et al., 2013]. There is strong D1 MSN reduction after the first execution, while D2 MSNs show progressive reduction after extended training [Sommer et al., 2014]. The direct path is essential for the initiation of attended actions, while execution of learned sequences mostly uses the indirect path [Agnoli et al., 2013].

D1 is associated with reward prediction error, subjective reward value [Schultz, 2016], risk taking [Rutledge et al., 2015], motivation, effort, perseverance, and vigor [Salamone and Correa, 2012]. However, it is also released in aversive events [Bromberg-Martin et al., 2010], including aggression [Anstrom et al., 2009]. The acting role of DA explains these seemingly paradoxical facts. Hence, the common description of DA's role as supporting reward is inaccurate. This description better suits BEND.

Execution. AChRs are expressed in both excitatory and coordination neurons. nAChRs and $\mathrm{mAChRs}$ desensitize quickly and slowly (respectively), according with mediation of competition and automated responses (nAChRs) and of acute responses (mAChRs). Alpha7 nAChR disinhibits apical pyramidal dendrites [Letzkus et al., 2011] exciting DCN VIPs. mAChRs excite the indirect BG path and suppress the direct path [Ding et al., 2010], supporting execution and suppressing the candidates mode.

Planning. SER promotes planning by suppressing innate responses ('emotions') and executing responses via SER1R, exciting the DM network via SER2R and SER3R ${ }^{D}$, and exciting event memory areas via SER4/6. SER2 agonists such as psilocybin and LSD induce hallucinations via excessive excitation of the DM network, which amplifies imagery 
without suppressing responses. Selective serotonin reuptake inhibitors (SSRIs) are used to counter depression, by suppressing negative emotions, reducing DM urgency, and promoting coping via planning.

Automaticity. D2R favors the response network [Gee et al., 2012], briefly suppressing and then exciting the cell. Small amounts of SER facilitate motoneurons via dendritic SER2 and SER7 [Perrier and Cotel, 2015]. High-affinity alpha4beta2 nAChRs are mostly located presynaptically on pyramid-targeting IINs, supported automated execution.

Success. BEND is released by HT arcuate and some NST neurons and by the pituitary, and acts via suppressive mu-opioid receptors (MORs). BEND excites DA neurons to promote acting, both directly [Fields and Margolis, 2015] and by suppressing GABAergic RMTg [Jhou et al., 2009] and STR neurons (striosomes) that suppress DA neurons ${ }^{D}$. It also suppresses CNs [Taki et al., 2000] to de-synchronize executing quacia, and suppresses the intercalated masses ${ }^{D}$, small GABAergic amygdala nuclei that allow PFC to suppress innate responses.

OT induces relaxation ${ }^{D}$ by suppressing stress [Uvnäs-Moberg et al., 2014].

For evidence supporting the roles of $\mathrm{MCH}$ in interaction, DYN in withdrawal, ENK in locomotion, nociceptin and SP in fight/pain, CRH in extended alerts, and GCs, ECBs and DHEA in energy management, $\mathrm{see}^{D}$.

\subsection{Experimental phenomena}

Here we discuss well-known phenomena explained by R17 and supporting it. Other phenomena, including the attentional blink, repetition blindness, inattentional blindness, Stroop effect, and attentional cueing, are discussed in ${ }^{D}$.

Electroencephalography (EEG). Cortical pyramidal neurons have a main dendritic branch oriented perpendicular to the cortical sheet. EEG mostly reflects the relative distribution of neuronal activity between the superficial and deep layers [Kotchoubey, 2006] ${ }^{8}$. When EEG measurements are synchronized with behavioral responses and averaged to reduce noise, clear negativities and positivities called event-related potentials (ERPs) are obtained. Well-known ERPs include the N170 (face recognition), MMN (mismatch negativity, usually auditory), N2 (visual attention), ERN (error-related negativity), P3b (P300, frontal response to objects), ELAN (early left-anterior negativity, local language syntax), N400 (response to words), and LDAEP (loudness dependent auditory evoked potential).

ERPs provide strong evidence for competition and the roles of the superficial and deep networks. Negative ERPs reflect the peak of DM network activity, while positive ERPs correspond to response network activity during and after competition. Indeed, ERP negativity size correlates with the amount of surprise: a larger language N400 reflects a larger semantic surprise. Larger positivities reflect increased competition, e.g., the P3b component is larger for rare stimuli [Verleger, 2008].

The EEG also shows oscillations in various frequency bands ${ }^{D}$. Oscillations are clearest when execution or rest are not interrupted, and show event-related desynchronization after surprises. This supports the synchronizing role of the ECN during sustained execution.

Planning as a Q process is supported by EEG sharp wave ripples (SWRs), which originate at the hippocampus, propagate to many brain areas, and are involved in navigation planning [Roumis and Frank, 2015]. Moreover, direct recordings show that animals imagine action scenarios in CA1 before executing them [Pfeiffer and Foster, 2013].

\footnotetext{
${ }^{8}$ This is true only coarsely, since cortex is folded and electrodes are located on the scalp.
} 
Imaging. Functional MRI (fMRI) measures blood-oxygen-level dependent (BOLD) contrasts. High BOLD mostly reflects TD flow [Cardoso et al., 2012], highest during candidate activation.

Repetition suppression (RS). When an event is repeated several times, its measurable effects are decreased (RS or adaptation). BOLD shows clear RS. This supports the R process, because when an event is repeated, the acute response to it decreases.

Mirror neurons (MNs). MNs [Rizzolatti and Sinigaglia, 2008] are directly recorded neurons that fire both when an animal performs an action and when it observes a similar action. R17 views MNs as part of the DM response during action observation and as neurons mobilized by action nodes during execution. Since the actions are similar, there are many features shared by the two situations, so there should be many neurons excited in both situations.

$\mathrm{MN}$ facts fit the DM network. They are easily found in brain recordings, exist in many brain areas, are mostly in the DM network, and can predictively activate before an action [Kilner et al., 2004] ${ }^{D}$.

Wait, working memory (WM). Animal can wait for a specific event (condition) to occur, after which they execute a planned action. Both the condition and the planned action are implemented as Q process goals ${ }^{D}$. M1 and thalamus neurons show bidirectional persistent activity during a task delay period, which predicts the specific response [Guo et al., 2017]. When there is uncertainty about the condition's timing, activation is of the DM network. As uncertainty diminishes, activation moves to the prediction network.

Usage of the DM network during wait is strongly supported by slowly increasing EEG negativities during event anticipation (readiness potential, contingent negative variation [Brunia et al., 2012]). D1Rs are essential for correct timing of learned conditions [Narayanan, 2016], supporting their role in predictions and goal-setting.

In WM experiments, the animal is shown an object (study item) that then disappears, and needs to execute a specific movement when this specific object reappears. During the delay period, study items are not present yet are actively kept, and this detachment from reality is sometimes viewed as fundamental to high-level cognition. R17 views WM as similar to wait, the difference being that none of the condition's features is externally present.

Since the timing of object reappearance is uncertain, delay period activity is in the superficial networks, as evidenced by single unit recording [Arnsten, 2013] and sustained BOLD and EEG negativity [Perez et al., 2012]. Thus, WM is a DM planning mode. A sustained anterior negativity (SAN) increases with the number of auditory items in memory, leveling at 4+ items [Alunni-Menichini et al., 2014].

There are well-known limits $(7 \pm 2,4)$ on the number of objects that animals can maintain in WM. Since each object needs to maintain a separate quax mapping it to the response, and since there is high overlap between quax neurons, having multiple objects increases the probability of quax collapse ${ }^{D}$.

Place cells. The hippocampus contains place cells, active when the animal reaches specific locations. The hippocampus shows a dominant EEG theta rhythm $(4-10 \mathrm{~Hz})$ during rodent activity. Under certain conditions (especially uniform running) place cell firing exhibits theta phase precession (TPP), i.e., the cell fires once in every cycle of the theta rhythm, firing earlier in the cycle in consecutive cycles [Malhotra et al., 2012].

The R17 view is that place cells are action goals. Indeed, they are most precise at CA1, the prediction network field. Attaining a goal activates the response network so yields an 
EEG positivity, the theta peak. Peaks are equidistant because running is at constant speed and place cells are approximately evenly spaced [Cei et al., 2014].

When the animal reaches the place serving as an action goal, the attained goal triggers the next action, which opens another place goal. Since it is faster to reactivate a neuron than to attain a motor goal, the next time the cell fires should be earlier in the theta cycle, including for subsequent firings. This explains TPP, which supports the Q process account of action sequences.

Our accounts explains why there are species (e.g., bats) that have place cells but show a theta rhythm that is qualitatively different from that of rodents [Ulanovsky and Moss, 2007]. Place cells are due to meaningful events, while theta arises during subsequent execution in which place cells are goals. Bats can yield theta while sensing the environment using echolocation rather than locomotion.

Language. Predictions are widely exhibited in language [DeLong et al., 2014] (e.g., the N400). SANs start right after hearing a word that generates focused expectations, and increase until the predicted word arrives [Li et al., 2017].

Cross-modal lexical priming shows that the meaning of a verb continues to be active only as long as its clause is not closed [De Goede et al., 2009]. This supports hierarchical mobilization: the meaning of a verb continues to prime related words because the nodes that have activated it still actively excite it. It also supports the existence of syntax nodes having a similar nature to short motor action plan nodes, since mobilization stops when the syntactic unit (the clause) terminates.

Semantic conflict beyond the single word yields increased competition in the response network in meaning areas. For example, upon hearing 'The boy kicked...', a prediction for what was kicked is formed, but if the next word is 'by' it activates new competing action nodes. Indeed, the P600 ERP is larger when an initial interpretation of the sentence needs to be revised ${ }^{D}$.

Magnetoencephalography in human speakers shows that sensory predictions do not track movement variability across repetitions of the same motor task, and hence represent the sensory goal, not a copy of the motor command (efference copy) [Niziolek et al., 2013].

Motor neurons controlling various effectors bidirectionally affect language processing in an effector-specific manner [Schomers et al., 2015]. This and other embodied cognition results are easily explained by reciprocal mobilization of motor, task and word nodes.

Language comprehension is the language analogue of action observation. Indeed, there is substantial evidence that production and comprehension share syntax areas and predictive mechanisms ${ }^{D}$.

The claim that language depends on recursion has received much publicity. We report psycholinguistic experiments showing that the brain does not use $\operatorname{recursion}^{D}$.

\subsection{The immune system}

The general $\mathrm{R}$ process is basically identical to the immune response [Paul, 2013]. The comparison is not a metaphor; the two systems undergo the same process and cooperate in real time during the evolution of its stages.

Like R17, immunity distinguishes between innate, adaptive, learned and acute responses. Its alerts are supported by inflammatory cytokines and antigen presenting cells that present short amino acids (features) of the pathogen and move to lymph nodes (BU) to recruit adaptive immune cells. The DM mode is implemented by candidate $\mathrm{B}$ and $\mathrm{T}$ cells that respond to these features by proliferation and migration towards the alerting tissue (TD). These cells have been trained to respond to features during thymus development, and yield a coarse 
response, inflammation. Anti-inflammatory cytokines mediate competition to sharpen inflammation into a focused response, which is learned for future responses (allowing vaccination).

The immune system and the brain are the two major organism systems that address sensory stimuli. The fact that the immune system is known to be governed by an R process lends strong support to R17. The similarities between the two processes even extend to some lower-level details, e.g., the role of glucocorticoids in quenching the acute response, and the anti-inflammatory effect of ACh when transitioning to focused responses [Pavlov and Tracey, 2015].

\section{Discussion}

This paper suggests that brain function, widely viewed as a mystery, can be explained using a single simple process. Although this may be surprising, there is no reason why the brain cannot be explained as other biological organs have been, especially considering the vast amount of experimental evidence generated during the last 50 years.

As a general scientific theory, R17 has several positive properties. First, it is comprehensive, accounting for virtually all of the relevant important behavioral facts (as explained, learning and disorders are presented elsewhere). Second, it is supported by converging evidence from multiple modalities. Third, it is not contradicted by any existing evidence. Fourth, it is conceptually simple. Finally, it is mechanistically detailed, allowing the formulation of theory predictions.

As a brain theory, R17 has the positive property of showing how high-level constructs emerge from low-level biological processes. High-level cognitive theories cannot make detailed theory predictions, while low-level descriptions are not sufficiently explanatory.

Previous work. Virtually all of the central notions in R17 are novel, not having been recognized by previous work. These include the $\mathrm{R}$ process, the $\mathrm{N}$ process, the management of $\mathrm{R}$ modes by Rgens, the existence and roles of $\mathrm{R}$ networks, and the $\mathrm{Q}$ process account of action sequences, language, and thought.

Strikingly, R17 dramatically differs from the accepted views in several central topics, most notably the BG, mirror neurons, language, optimal motor control, DA, SER, MCH and opioids. Highlighting three examples, the accepted views of the BG are that the direct path supports movement while the indirect path stops movement, or that the indirect path selects between competing actions [Graybiel and Mink, 2009]. However, these are contradicted by evidence (above). DA is viewed as the brain's reward agent, but it is also released in aversive events and aggression. SER is viewed as inducing happiness, but it suppresses emotions $^{D}$. In all of these, the R17 account accords with the evidence and seamlessly integrates with the overall theory.

Currently there exists no comprehensive mechanistic theory of the brain. Some existing accounts overlap with some aspects of R17 but address specific areas without sufficient detail or generalization. These include biased competition [Desimone and Duncan, 1995], predictive coding [Adams et al., 2013], dual-process theories [Kahneman, 2011], and classical notions such as Hebb's cell assemblies, affordances, the ideomotor principle, efference copy (corollary discharge), and the action perception cycle ${ }^{D}$.

Falsification and theory predictions. R17 gives a precise description of the role of Rgens and of the sequence of neuronal activation in various execution modes. It is thus inherently falsifiable and can be used to derive a large number of theory predictions. Any technique 
that measures neurons of a specific type and discovers a consistently different activation sequence would refute the relevant parts of R17.

\section{Conclusion}

This paper is admittedly ambitious, presenting a simple theory that claims to answer most of the fundamental questions about cognition and the brain. Yet, the theory is strongly supported by the available facts and by its simplicity. I hope that the paper inspires other researchers to develop comprehensive brain theories, to the advancement of science.

\section{References}

[Adams et al., 2013] Adams, R. A., Shipp, S., and Friston, K. J. (2013). Predictions not commands: active inference in the motor system. Brain Structure and Function, 218(3):611-643.

[Adolphs, 2015] Adolphs, R. (2015). The unsolved problems of neuroscience. Trends in cognitive sciences, 19(4):173-175.

[Agnoli et al., 2013] Agnoli, L., Mainolfi, P., Invernizzi, R. W., and Carli, M. (2013). Dopamine D1-like and D2-like receptors in the dorsal striatum control different aspects of attentional performance in the five-choice serial reaction time task under a condition of increased activity of corticostriatal inputs. Neuropsychopharmacology, 38(5):701714.

[Alunni-Menichini et al., 2014] Alunni-Menichini, K., Guimond, S., Bermudez, P., Nolden, S., Lefebvre, C., and Jolicoeur, P. (2014). Saturation of auditory short-term memory causes a plateau in the sustained anterior negativity event-related potential. Brain research, 1592:55-64.

[Anstrom et al., 2009] Anstrom, K. K., Miczek, K. A., and Budygin, E. A. (2009). Increased phasic dopamine signaling in the mesolimbic pathway during social defeat in rats. Neuroscience, 161(1):3-12.

[Arnsten, 2013] Arnsten, A. (2013). The neurobiology of thought: the groundbreaking discoveries of Patricia Goldman-Rakic 1937-2003. Cerebral Cortex, 23(10):2269-2281.

[Bromberg-Martin et al., 2010] Bromberg-Martin, E. S., Matsumoto, M., and Hikosaka, O. (2010). Dopamine in motivational control: rewarding, aversive, and alerting. Neuron, 68(5):815-834.

[Brunia et al., 2012] Brunia, C., van Boxtel, G., Böcker, K., Kappenman, E., and Luck, S. (2012). Negative slow waves as indices of anticipation: The bereitschaftspotential, the contingent negative variation, and the stimulus-preceding negativity. The Oxford handbook of event-related potential components, pages 189-207.

[Cardoso et al., 2012] Cardoso, M. M., Sirotin, Y. B., Lima, B., Glushenkova, E., and Das, A. (2012). The neuroimaging signal is a linear sum of neurally distinct stimulus-and task-related components. Nature neuroscience, 15(9):1298-1306. 
[Cei et al., 2014] Cei, A., Girardeau, G., Drieu, C., El Kanbi, K., and Zugaro, M. (2014). Reversed theta sequences of hippocampal cell assemblies during backward travel. $\mathrm{Na}$ ture neuroscience, 17(5):719-724.

[Craig, 2014] Craig, A. (2014). How do you feel?: an interoceptive moment with your neurobiological self. Princeton University Press.

[Cui et al., 2013] Cui, G., Jun, S. B., Jin, X., Pham, M. D., Vogel, S. S., Lovinger, D. M., and Costa, R. M. (2013). Concurrent activation of striatal direct and indirect pathways during action initiation. Nature, 494(7436):238-242.

[De Goede et al., 2009] De Goede, D., Shapiro, L. P., Wester, F., Swinney, D. A., and Bastiaanse, R. (2009). The time course of verb processing in Dutch sentences. Journal of psycholinguistic research, 38(3):181-199.

[DeLong et al., 2014] DeLong, K. A., Troyer, M., and Kutas, M. (2014). Pre-processing in sentence comprehension: Sensitivity to likely upcoming meaning and structure. Language and Linguistics Compass, 8(12):631-645.

[Desimone and Duncan, 1995] Desimone, R. and Duncan, J. (1995). Neural mechanisms of selective visual attention. Annual review of neuroscience, 18(1):193-222.

[Ding et al., 2010] Ding, J. B., Guzman, J. N., Peterson, J. D., Goldberg, J. A., and Surmeier, D. J. (2010). Thalamic gating of corticostriatal signaling by cholinergic interneurons. Neuron, 67(2):294-307.

[Fields and Margolis, 2015] Fields, H. L. and Margolis, E. B. (2015). Understanding opioid reward. Trends in neurosciences, 38(4):217-225.

[Gee et al., 2012] Gee, S., Ellwood, I., Patel, T., Luongo, F., Deisseroth, K., and Sohal, V. S. (2012). Synaptic activity unmasks dopamine D2 receptor modulation of a specific class of layer V pyramidal neurons in prefrontal cortex. The Journal of Neuroscience, 32(14):4959-4971.

[Goldman-Rakic et al., 1990] Goldman-Rakic, P., Lidow, M., and Gallager, D. (1990). Overlap of dopaminergic, adrenergic, and serotoninergic receptors and complementarity of their subtypes in primate prefrontal cortex. The Journal of Neuroscience, 10(7):21252138.

[Graybiel and Mink, 2009] Graybiel, A. and Mink, J. (2009). The basal ganglia and cognition. In Gazzaniga, M., editor, The Cognitive Neurosciences IV. MIT Press, Cambridge, MA.

[Grillner, 2014] Grillner, S. (2014). Megascience efforts and the brain. Neuron, 82(6):1209-1211.

[Guo et al., 2017] Guo, Z. V., Inagaki, H. K., Daie, K., Druckmann, S., Gerfen, C. R., and Svoboda, K. (2017). Maintenance of persistent activity in a frontal thalamocortical loop. Nature.

[Jhou et al., 2009] Jhou, T. C., Geisler, S., Marinelli, M., Degarmo, B. A., and Zahm, D. S. (2009). The mesopontine rostromedial tegmental nucleus: a structure targeted by the lateral habenula that projects to the ventral tegmental area of Tsai and substantia nigra compacta. Journal of Comparative Neurology, 513(6):566-596. 
[Kahneman, 2011] Kahneman, D. (2011). Thinking, fast and slow. Macmillan.

[Karnani et al., 2016] Karnani, M. M., Jackson, J., Ayzenshtat, I., Sichani, A. H., Manoocheri, K., Kim, S., and Yuste, R. (2016). Opening holes in the blanket of inhibition: localized lateral disinhibition by VIP interneurons. Journal of Neuroscience, 36(12):3471-3480.

[Kilner et al., 2004] Kilner, J. M., Vargas, C., Duval, S., Blakemore, S.-J., and Sirigu, A. (2004). Motor activation prior to observation of a predicted movement. Nature neuroscience, 7(12):1299.

[Koch and Marcus, 2014] Koch, C. and Marcus, G. (2014). Neuroscience in 2064: a look at the last century. In Marcus, G. and Freeman, J., editors, The Future of the Brain: Essays by the World's Leading Neuroscientists. Princeton University Press.

[Kotchoubey, 2006] Kotchoubey, B. (2006). Event-related potentials, cognition, and behavior: a biological approach. Neuroscience \& Biobehavioral Reviews, 30(1):42-65.

[Letzkus et al., 2015] Letzkus, J. J., Wolff, S. B., and Lüthi, A. (2015). Disinhibition, a circuit mechanism for associative learning and memory. Neuron, 88(2):264-276.

[Letzkus et al., 2011] Letzkus, J. J., Wolff, S. B., Meyer, E. M., Tovote, P., Courtin, J., Herry, C., and Lüthi, A. (2011). A disinhibitory microcircuit for associative fear learning in the auditory cortex. Nature, 480(7377):331-335.

[Li et al., 2017] Li, X., Zhang, Y., Xia, J., and Swaab, T. (2017). Internal mechanisms underlying anticipatory language processing: Evidence from event-related-potentials and neural oscillations. Neuropsychologia, 102:70-81.

[Malhotra et al., 2012] Malhotra, S., Cross, R. W., and van der Meer, M. A. (2012). Theta phase precession beyond the hippocampus. Reviews in the neurosciences, 23(1):39-65.

[Narayanan, 2016] Narayanan, N. S. (2016). Ramping activity is a cortical mechanism of temporal control of action. Current opinion in behavioral sciences, 8:226-230.

[Niziolek et al., 2013] Niziolek, C. A., Nagarajan, S. S., and Houde, J. F. (2013). What does motor efference copy represent? evidence from speech production. Journal of Neuroscience, 33(41):16110-16116.

[Paul, 2013] Paul, W. (2013). Fundamental immunology, 7th edition.

[Pavlov and Tracey, 2015] Pavlov, V. A. and Tracey, K. J. (2015). Neural circuitry and immunity. Immunologic research, 63(1-3):38-57.

[Perez et al., 2012] Perez, V. B., Vogel, E. K., Luck, S., and Kappenman, E. (2012). What ERPs can tell us about working memory. The Oxford handbook of event-related potential components, pages 361-372.

[Perrier and Cotel, 2015] Perrier, J.-F. and Cotel, F. (2015). Serotonergic modulation of spinal motor control. Current opinion in neurobiology, 33:1-7.

[Pfeiffer and Foster, 2013] Pfeiffer, B. E. and Foster, D. J. (2013). Hippocampal place-cell sequences depict future paths to remembered goals. Nature, 497(7447):74-79. 
[Proulx et al., 2014] Proulx, C. D., Hikosaka, O., and Malinow, R. (2014). Reward processing by the lateral habenula in normal and depressive behaviors. Nature neuroscience, 17(9):1146-1152.

[Rappoport, 2018] Rappoport, A. (2018). The brain, explained: A comprehensive theory of brain function. doi: 10.20944/preprints201805.0137.

[Rizzolatti and Sinigaglia, 2008] Rizzolatti, G. and Sinigaglia, C. (2008). Mirrors in the brain: How our minds share actions and emotions. Oxford University Press, USA.

[Roumis and Frank, 2015] Roumis, D. K. and Frank, L. M. (2015). Hippocampal sharpwave ripples in waking and sleeping states. Current opinion in neurobiology, 35:6-12.

[Rutledge et al., 2015] Rutledge, R. B., Skandali, N., Dayan, P., and Dolan, R. J. (2015). Dopaminergic modulation of decision making and subjective well-being. Journal of Neuroscience, 35(27):9811-9822.

[Sakurai, 2014] Sakurai, T. (2014). The role of orexin in motivated behaviours. Nature Reviews Neuroscience, 15(11):719-731.

[Salamone and Correa, 2012] Salamone, J. D. and Correa, M. (2012). The mysterious motivational functions of mesolimbic dopamine. Neuron, 76(3):470-485.

[Sarter et al., 2016] Sarter, M., Lustig, C., Berry, A. S., Gritton, H., Howe, W. M., and Parikh, V. (2016). What do phasic cholinergic signals do? Neurobiology of Learning and Memory, 130:135-141.

[Schomers et al., 2015] Schomers, M. R., Kirilina, E., Weigand, A., Bajbouj, M., and Pulvermüller, F. (2015). Causal influence of articulatory motor cortex on comprehending single spoken words: Tms evidence. Cerebral cortex (new York, Ny: 1991), 25(10):3894-3902.

[Schultz, 2016] Schultz, W. (2016). Dopamine reward prediction-error signalling: a twocomponent response. Nature Reviews Neuroscience.

[Sherman and Guillery, 2013] Sherman, S. M. and Guillery, R. W. (2013). Functional connections of cortical areas: a new view from the thalamus. MIT Press.

[Sommer et al., 2014] Sommer, W. H., Costa, R. M., and Hansson, A. C. (2014). Dopamine systems adaptation during acquisition and consolidation of a skill. Frontiers in integrative neuroscience, 8:87.

[Taki et al., 2000] Taki, K., Kaneko, T., and Mizuno, N. (2000). A group of cortical interneurons expressing $\mu$-opioid receptor-like immunoreactivity: a double immunofluorescence study in the rat cerebral cortex. Neuroscience, 98(2):221-231.

[Tejeda et al., 2012] Tejeda, H., Shippenberg, T., and Henriksson, R. (2012). The dynorphin/ $\kappa$-opioid receptor system and its role in psychiatric disorders. Cellular and Molecular Life Sciences, 69(6):857-896.

[Ulanovsky and Moss, 2007] Ulanovsky, N. and Moss, C. F. (2007). Hippocampal cellular and network activity in freely moving echolocating bats. Nature neuroscience, 10(2):224. 
[Uvnäs-Moberg et al., 2014] Uvnäs-Moberg, K., Handlin, L., and Petersson, M. (2014). Self-soothing behaviors with particular reference to oxytocin release induced by nonnoxious sensory stimulation. Frontiers in psychology, 5:1529-1529.

[Veening et al., 2015] Veening, J., De Jong, T., Waldinger, M., Korte, S., and Olivier, B. (2015). The role of oxytocin in male and female reproductive behavior. European journal of pharmacology, 753:209-228.

[Verleger, 2008] Verleger, R. (2008). P3b: towards some decision about memory. Clinical Neurophysiology, 119(4):968-970. 